PROCEEDINGS OF THE

AMERICAN MATHEMATICAL SOCIETY

Volume 139, Number 4, April 2011, Pages 1469-1472

S 0002-9939(2010)10558-0

Article electronically published on August 27, 2010

\title{
NONPOSITIVELY CURVED HERMITIAN METRICS ON PRODUCT MANIFOLDS
}

\author{
CHENGJIE YU
}

(Communicated by Jianguo Cao)

\begin{abstract}
In this article, we classify all the Hermitian metrics on a complex product manifold $M=X \times Y$ with nonpositive holomorphic bisectional curvature. It is a generalization of a result by Zheng.
\end{abstract}

In this article, using a trick as in Tam-Yu 1, we prove the following generalization of the result in Zheng [2].

Theorem 0.1. Let $M=X \times Y$ with $X$ and $Y$ both compact complex manifolds. Let $\phi_{1}, \phi_{2}, \cdots, \phi_{r}$ be a basis of $H^{1,0}(X)$ and $\psi_{1}, \psi_{2}, \cdots, \psi_{s}$ be a basis of $H^{1,0}(Y)$. Then, for any Hermitian metric $h$ on $M$ with nonpositive holomorphic bisectional curvature,

$$
\omega_{h}=\pi_{1}^{*} \omega_{h_{1}}+\pi_{2}^{*} \omega_{h_{2}}+\rho+\bar{\rho},
$$

where $h_{1}$ and $h_{2}$ are Hermitian metrics on $X$ and $Y$ with nonpositive holomorphic bisectional curvature respectively, $\pi_{1}$ and $\pi_{2}$ are natural projections from $M$ to $X$ and from $M$ to $Y$ respectively, and

$$
\rho=\sqrt{-1} \sum_{k=1}^{r} \sum_{l=1}^{s} a_{k l} \phi_{k} \wedge \psi_{l}
$$

where the $a_{k l}$ 's are complex numbers.

Before the proof of Theorem 0.1 we need the following lemma.

Lemma 0.2. Let $X^{m}$ and $Y^{n}$ be two compact complex manifolds. Let $\phi_{1}, \phi_{2}, \cdots, \phi_{r}$ be a basis of $H^{1,0}(X)$ and $\psi_{1}, \psi_{2}, \cdots, \psi_{s}$ be a basis of $H^{1,0}(Y)$. Let

$$
\rho=\rho_{i j}(x, y) d x^{i} \wedge d y^{j}
$$

be a global holomorphic two form on $X \times Y$, where $\left(x^{1}, x^{2}, \cdots, x^{m}\right)$ is a local holomorphic coordinate of $X$ and $\left(y^{1}, y^{2}, \cdots, y^{n}\right)$ is a local holomorphic coordinate of $Y$.

Received by the editors February 28, 2010 and, in revised form, April 24, 2010

2000 Mathematics Subject Classification. Primary 53B25; Secondary 53C40.

Key words and phrases. Complex products, Kähler manifolds, bisectional curvature, nonpositive curvature.

This research was partially supported by the National Natural Science Foundation of China (10901072) and GDNSF (9451503101004122).

(C)2010 American Mathematical Society Reverts to public domain 28 years from publication 
Then

$$
\rho=\sum_{k=1}^{r} \sum_{l=1}^{s} a_{k l} \phi_{k} \wedge \psi_{l},
$$

where the $a_{k l}$ 's are complex numbers.

Proof. Fix a local holomorphic coordinate $\left(y^{1}, y^{2}, \cdots, y^{n}\right)$ of $Y$. It is clear that

$$
\theta_{j}=\sum_{i=1}^{m} \rho_{i j}(x, y) d x^{i}
$$

is a global homomorphic 1 -form on $X \times\{y\}$. Then

$$
\theta_{j}=\sum_{k=1}^{r} b_{k j}(y) \phi_{k},
$$

where the $b_{k j}$ 's are local homomorphic functions on $Y$.

It is clear that

$$
\sum_{j=1}^{n} b_{k j}(y) d y^{j}
$$

is a global holomorphic 1-form on $Y$ for each $k$. So,

$$
\sum_{j=1}^{n} b_{k j}(y) d y^{j}=\sum_{l=1}^{s} a_{k l} \psi_{l},
$$

where the $a_{k l}$ 's are complex numbers. Therefore

$$
\rho=\sum_{j=1}^{n} \theta_{j} \wedge d y^{j}=\sum_{k}^{r} \sum_{l=1}^{s} a_{k l} \phi_{k} \wedge \psi_{l} .
$$

Proof of Theorem 0.1 . Let $\left(z^{m+1}, \cdots, z^{m+n}\right)$ be a local holomorphic coordinate of $Y$ at $q$. Then, it is clear that

$$
h_{\alpha \bar{\alpha}}(x, q)
$$

is a positive function on $X \times\{q\}$, where $m+1 \leq \alpha \leq m+n$.

Let $\Delta$ be the complex Laplacian on $X \times\{q\}$ and let $\left(z^{1}, z^{2}, \cdots, z^{m}\right)$ be a holomorphic coordinate of $X$ such that

$$
h_{i \bar{j}}(x, q)=\delta_{i \bar{j}}
$$

with $1 \leq i, j \leq m$. Then

$$
\Delta h_{\alpha \bar{\alpha}}(x, q)=\sum_{i=1}^{m} \partial_{i} \partial_{\bar{i}} h_{\alpha \bar{\alpha}}=-\sum_{i=1}^{m} R_{\alpha \bar{\alpha} \bar{i} \bar{i}}+\sum_{i=1}^{m} h^{\bar{b} a} \partial_{i} h_{\alpha \bar{b}} \partial_{\bar{i}} h_{a \bar{\alpha}} \geq 0,
$$

with $1 \leq a, b \leq n+m$. By the maximum principle, $h_{\alpha \bar{\alpha}}(x, q)$ is a constant function. Hence

$$
\partial_{i} h_{\alpha \bar{b}}=0 .
$$

Interchanging the roles of $X$ and $Y$ in the above, we get

$$
\partial_{\alpha} h_{i \bar{b}}=0 .
$$

By (0.7), we know that

$$
\partial_{i} h_{\alpha \bar{\beta}}=0
$$


for any $m+1 \leq \alpha, \beta \leq n+m$. So, $h_{\alpha \bar{\beta}}$ is independent of the $z^{i}$ 's. Then, $h_{\alpha \bar{\beta}}$ is a Hermitian metric on $Y$. It is clear that $h_{\alpha \bar{\beta}}$ as a Hermitian metric on $Y$ is of nonpositive holomorphic bisectional curvature since holomorphic bisectional curvature deceases on complex submanifolds. We denote this metric by $h_{2}$.

Similarly, by (0.8), $h_{i \bar{j}}$ is a Hermitian metric on $X$ with nonpositive holomorphic bisectional curvature. We denote it by $h_{1}$.

By (0.8) and (0.7), we have

$$
\partial_{\alpha} h_{i \bar{\beta}}=0 \text { and } \partial_{\bar{i}} h_{j \bar{\alpha}}=0 \text {. }
$$

So, the form $h_{i \bar{\alpha}} d z^{i} \wedge d z^{\bar{\alpha}}$ is a holomorphic two form on $M_{1} \times \overline{M_{2}}$, where $\overline{M_{2}}$ is the complex conjugate of $M_{2}$. By the lemma above, we know that

$$
h_{i \bar{\alpha}} d z^{i} \wedge d z^{\bar{\alpha}}=\sum_{k=1}^{q_{1}} \sum_{l=1}^{q_{2}} a_{k l} \phi_{k} \wedge \bar{\psi}_{l} .
$$

Hence, we get the conclusion.

As in Zheng [2, we have the following consequence of the theorem.

\section{Corollary 0.1.}

$$
\operatorname{codim}_{\mathbb{R}}\left(\mathcal{H}\left(M_{1}\right) \times \mathcal{H}\left(M_{2}\right), \mathcal{H}\left(M_{1} \times M_{2}\right)\right)=2 h^{1,0}\left(M_{1}\right) \cdot h^{1,0}\left(M_{2}\right),
$$

where $M_{1}, M_{2}$ are compact complex manifolds, and suppose that $\mathcal{H}\left(M_{i}\right) \neq \emptyset$ for $i=1,2$.

Proof. For any $h \in \mathcal{H}\left(M_{1} \times M_{2}\right)$, by the theorem, it has a unique decomposition,

$$
\omega_{h}=\pi_{1}^{*} \omega_{h_{1}}+\pi_{2}^{*} \omega_{h_{2}}+\rho+\bar{\rho},
$$

where $\rho=\sqrt{-1} \sum_{i=1}^{q_{1}} \sum_{j=1}^{q_{2}} a_{i j} \phi_{i} \wedge \bar{\psi}_{j}$ with $a_{i j} \in \mathbb{C}, h_{i} \in \mathcal{H}\left(M_{i}\right)$. So, we get a map

$$
\mathcal{H}\left(M_{1} \times M_{2}\right) \rightarrow M\left(q_{1} \times q_{2} ; \mathbb{C}\right), h \mapsto\left(a_{i j}\right)_{q_{1} \times q_{2}}
$$

It is clear $\mathbb{R}^{+}$-linearly. (Note that $\mathcal{H}\left(M_{1} \times M_{2}\right)$ is a convex cone.) So, it induces a linear map of real vector spaces,

$$
\Psi:\left\langle\mathcal{H}\left(M_{1} \times M_{2}\right)\right\rangle_{\mathbb{R}} \rightarrow M\left(q_{1} \times q_{2} ; \mathbb{C}\right) .
$$

It is clear that

$$
\operatorname{ker} \Psi=\left\langle\mathcal{H}\left(M_{1}\right) \times \mathcal{H}\left(M_{2}\right)\right\rangle_{\mathbb{R}} .
$$

Moreover, let $E_{k l}=\left(a_{i j}\right)$ be such that $a_{i j}=\delta_{i k} \delta_{j l}$. Note that

$$
\begin{aligned}
& \pi_{1}^{*} \omega_{h_{1}}+\pi_{2}^{*} \omega_{h_{2}}+\sqrt{-1}\left(\phi_{k}+\psi_{l}\right) \wedge \overline{\left(\phi_{k}+\psi_{l}\right)} \\
= & {\left[\pi_{1}^{*} \omega_{h_{1}}+\sqrt{-1} \phi_{k} \wedge \bar{\phi}_{k}\right]+\left[\pi_{1}^{*} \omega_{h_{2}}+\sqrt{-1} \psi_{l} \wedge \bar{\psi}_{l}\right]+\sqrt{-1} \phi_{k} \wedge \bar{\psi}_{l}+\sqrt{-1} \psi_{l} \wedge \bar{\phi}_{k} . }
\end{aligned}
$$

So, $E_{k l}$ is in the image of $\Psi$. Similarly, $\sqrt{-1} E_{k l}$ is also in the image of $\Psi$. Therefore, $\Psi$ is surjective. By the dimension theorem in linear algebra, we get the identity. 


\section{REFERENCES}

[1] Tam, Luen-Fai; Yu, Chengjie. Complex Product Manifolds and Bounds of Curvature, to appear in Asian Journal of Mathematics.

[2] Zheng, F., Non-positively curved Kähler metrics on product manifolds, Ann. of Math. 137 (1993), 671-673. MR1217351 (94k:53083)

Department of Mathematics, Shantou University, Shantou, Guangdong, 515063 PeoPle's Republic of China

E-mail address: cjyu@stu.edu.cn 